\title{
A Study on Improving the Performance of TCP and Various Design Issues in Manets
}

\author{
Ramesh Pusuluri and Reena Aggarwal \\ Department of Electronics and Communication, Jalandhar-Delhi G.T. Road, National Highway 1, \\ Phagwara, Punjab - 144411, India; rameshpusuluri999@gmail.com, reena.16120@lpu.co.in
}

\begin{abstract}
Objective: To improve the performance of Transmission Control Protocol (TCP)by reducing packet loss, transmission errors, Round Trip Delay Time, optimizing the buffer size and using the energy efficient routing protocol. Methods: To achieve good performance, a feedback scheme is proposed which is called Feedback based TCP or TCP-F, so that the source can distinguish between Route Failure Notification (RFN) packet, allowing it to freeze its timers and stop sending packets. When the route is re-established, the source is informed through a Route Re-establishment Notification (RRN) packet, upon which it resumes by unfreezing timers and continuing packet transmissions. Findings: The performance of TCP on ad-hoc networks without and with feedback will be compared. The network performance will be determined in terms of throughput, time delay and routing overheads for different number of nodes. Improvements: It is assumed that in the event of route failures, as the route re-establishment time increases, the use of feedback provides significant gains in throughput as well as savings in unnecessary packet transmissions.
\end{abstract}

Keywords: MANETs, Packet Loss, TCP Performance, Throughput, Transmission Errors

\section{Introduction}

Wireless networks are very important in communication equipment. At present they are used in various industrial, military applications and networks which are personalized. The primary difference between wired network and wireless networks was the channel of communication. In wired networks physical medium is present, where as in case of wireless networks, there is no physical medium. Wireless networks are very popular networks in wide variety of applications by considering some of the following factors: reliability, easy to install, bandwidth, operating cost, security, amount of power required to operate and network performancef, All these networks rely on infrastructures which are fixed. Some of the most common wireless networks which based on infrastructure are cellular networks, Radio Detection And Ranging(RADAR), cordless telephone, Wi-MAX, Wi-Fi, Satellite communication, Microwave communication and Cordless Telephone etc.
A Mobile Ad Hoc Network (MANET) is obtained without the need of centralized structures by a group of mobile nodes which forms an ad-hoc network. These networks are very advantageous for establishment because they are fit in an environment where the loss of infrastructure is acceptable and they are not that much effective in cost. The most famous IEEE 802.11 is a "WIFI" protocol can provide facilities of ad-hoc network at very low level even through when there is no point to access, whereas the mobile nodes were restricted to receive and send information. But, across the network, they does not route anything. These networks can function in a way such that they did not depend on anything else or they will connect to alarger network, e.g. Internet. These networks contains a set of using wireless links in which MHs uses to communicate among them, without using any other support of communication. These can be called as multihop wireless networks or mobile radio networks. When two or more MHsare in the range of each other if one mobile host can receive other mobile host's transmission.

* Author for correspondence 
Every Mobile Host works in a co-operative manner such that each acts as a router and it allows data packets which are directed towards other MHs through which they pass. One of the typical applications is a military operation or a recovering from a disasterf. These are not confined to specific kind of situations; in some other locations also the performance of these networks are far much better. For example, imagine a situation of people in a company with laptops, in a conference meeting in the absence of any kind of network services. They make their machines to work very by simply forming an ad hoc network. It is one of the examples where ad-hoc networks were used.

\section{Literature Survey}

Most of the TCPs usage has designed by taking wired networks into consideration. Because of congestion in the networks wired networks always experience data. In MANET the data losses are not due to congestion of the network and various reasons for the loss of data in Ad hoc networks like channel loss, Concealed and exposure of the stations, Asymmetrical path, breaking of the Network, Routing Failure and Restrictions on Power consumption.

To perform MANET more efficient and effective a hybrid mechanism was introduced to provide node cooperation among nodes in MANETs was presented byto over-come the misbehavior. It in-corporates only information on first-hand and functions based on Dynamic Source Routing (DSR) protocol. By collaborating with DSR, this method can identify malicious nodes in packet sending process.

To increase lifetime of network of the Mobile ad hoc network a new method called Efficient Power Aware Routing (EPAR) is proposed EPAR recognizes the capability of a node not by its unconsumed power which a battery holds, but also by the normal energy which was consumed in accurately sending packets of data using a desired link. Using the solution of min-max, EPAR chooses a route which has the capability to transmit largesized packets with minimum unconsumed capability of packet transmission.

In MANETs, to reduce the significance of intervention bounded by the selected node-disjoint multipath scheme two types of on-demand multipath routing protocols are introduced. One is Efficient Stable Disjoint Multipath Routing Protocol (ESDMR) and another one is Efficient Disjoint Multipath Routing Protocol (EDMR) which was presented The routing protocols which are proposed is having a more transmission rate and throughput which is very high.

To get elevated network utility and reduced power, a method was proposed which is cross-layer Rate-Effective Network Utility Maximization (RENUM) framework by considering wireless link losses, restrictions of rate outage probability and average delay. By some numerical simulations the proposed algorithm performs better than some other algorithms which provide maximum utility of the network without rate outage probability/average delay restrictions. The algorithm which was introduced has effective rate which is very high, power consumption which is very low and low delay.

To lessen unnecessary transmissions and loss of throughput in MANETs a feedback scheme was introduced. In this scheme when a broken route was found, source will get a packet which notifies the failure of the route, which allows it to stop its timers to work and it will discard transmitting data packets . Again when a route was constructed back, the source was instructed with a packet which notifies about the re-establishment of the route. After that the source will allow it's timers to resume and continue transmitting data packets. So, Feedback based TCP gives best performance for simulated results.

By extensive simulation it is found that the throughput of TCP declines significantly when the nodes move because of TCPs inability to notice the difference between congestion and Link Failure]. In order to compare the throughput in multi-hop networks he introduced a new concept called expected throughput. This metric showed how the performance of TCP can be improved using the technique of Explicit Link Failure Notification (ELFN).

In Long Term Evolution (LTE) network, if the process of communication is not controlled correctly then Handover may produce unusual degeneration of the communication. To mitigate these, three solutions were introduced first one is fast path switch, second one is handover prediction, and third one is active queue management. First two solutions avoid enormous delay in the delivery of packets in handover, where second one contains operating queue management and acts as the transport network. It is found from Simulation results that the solutions which are introduced have some merits. The performance of the TCP was raised significantly when the handover prediction is used with operating queue management. 


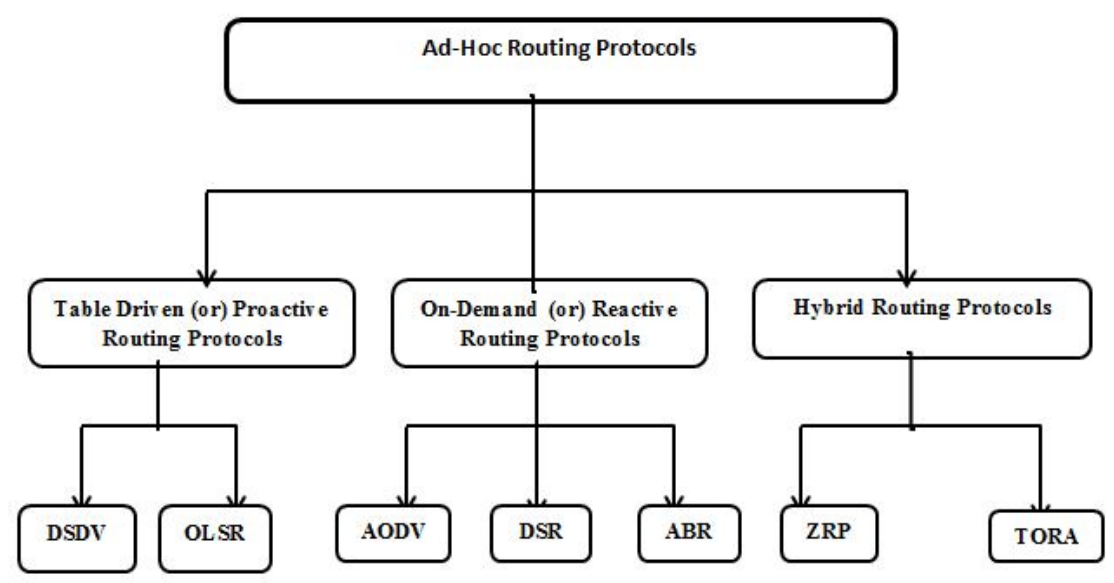

Figure 1. Classification of routing protocols in MANET.

\section{Routing Protocols in MANET}

MANETs are self-organizing multi-hop wireless networks, in which the network structure modifies dynamically. The main reason for this is due to node mobility. The nodes which are present in these networks access the channel in a random manner, collaborating in a confidential manner to fascinating themselves in multi-hop forwarding ? Each node in these network works like hosts and routers which send data to remaining nodes in network and receive data from other nodes in the network. MANETs doesn't contain any infrastructure support because, the destination node may be too much far from source node which is transmitting data packets. The classification of routing protocols is as shown in the Figure 1.

\subsection{Flat Routing Protocols}

These are classified into two types; 1$)$ proactive routing (table driven) protocols, 2) reactive (on-demand) routing protocols. In both of these protocol classes, each node plays an equal role in routing. Further they have been classified based on their design.

\subsection{Pro-Active Protocols}

They are also called as table driven protocols. These protocols preserve an updated network map. They steadily assess familiar routes and try to explore different routes. As the route is initially discovered, whenever a node need a path to a destination, or if a packet has to be delivered it will be done without any delay ${ }^{10}$. Otherwise, keeping the updated information needs more bandwidth which is not possible as there is finite battery power in MANETs and sometimes the information may be outdated. Protocols such as OLSR and Conventional Routing Schemes come in this pro-active class. Pro-active protocols which are pure are fairly not capable for ad hoc networks because nodes are highly mobile due to high traffic overhead which was produced due to the updating of information in the network. Otherwise, pure re-active protocols have high delays and high control traffic. Because of the following factors they are unfit for some applications.

\subsection{Re-Active Protocols}

These are on-demand protocols dissimilar to pro-active protocols and they start the process of discovering a new route when necessary. Whenever there is requirement of route from source node to the destination node, there is a search scheme which will be initiated globally ${ }^{10}$. It does not need any steady updates which were obtained from the network, as this leads to delays, as routes need to be found and they are not easy to access. Sometimes the required routes are present in the cache preserved by nodes. Since there is no need of routes explosion there is no extra delay. Re-active protocols members are AdHoc on Demand Multiple Path Distance Vector (AOMDV), Ad hoc On Demand Distance Vector (AODV), Temporally Ordered Routing Algorithm (TORA), and DSR.

\subsection{Hybrid Protocols}

They incorporate the merits of Re-active and Pro-active routing. It was slightly established on expectation that in MANETs most of the communication takes place among 
the nodes which are nearer. It is assumed that modifications of topology are important ${ }^{19}$. When a link failure occurs in some part of the network then the neighborhoods which are local were affected, but the nodes in the remaining part of the network remain unaffected.

\subsection{Hierarchical Routing Protocols (HRP)}

With the increase in the size of wireless networks, more overhead was evolved in MANETs by the flat routing protocolst So, a solution was found in order to overcome this. These are organized into Hierarchical State Routing (HSR), Cluster-head Gateway Switch Routing Protocol (CGSR), Zone Routing Protocol (ZRP), Landmark Ad Hoc Routing Protocol (LANMAR).

\subsection{Geographical Routing Protocols (GRP)}

These were of two types: 1). Actual geographic coordinates.

2). Fixed coordinate system with reference points.

One of the advantages of GRP is it inhibit searches which are in-network for required destination. The data control and data packets are sent in regular destination if geographical coordinates were acknowledged so that in the network the control overhead will be reduced One of the disadvantages is that in order to make the geographical routing protocols helpful, every node must have access to their coordinates which are located geographically all the time. The updating of routing must be performed in such a way that it should be rapid in contrast of the mobility rate of network to recognize the position based routing. Because in MANETs the positions of nodes may shift rapidly.

Examples of geographical routing protocols are: GeoCast (Geographic addressing and routing), Distance Routing Effect Algorithm for Mobility (DREAM) and Greedy Perimeter Stateless Routing (GPSR).

Table 1 showing the sub-classification of the routing protocols of the MANET with their description, examples and their disadvantages.

Table 1. Different routing protocols and their description

\begin{tabular}{|c|c|c|c|c|}
\hline $\begin{array}{l}\text { Name of Routing } \\
\text { protocol }\end{array}$ & $\begin{array}{l}\text { Sub } \\
\text { Classification }\end{array}$ & Description & Examples & Disadvantages \\
\hline \multirow[t]{3}{*}{$\begin{array}{l}\text { Flat Routing } \\
\text { Protocol }\end{array}$} & $\begin{array}{l}\text { Proactive } \\
\text { Protocol }\end{array}$ & $\begin{array}{l}\text { These Protocols try to main- } \\
\text { tain an up-to-date map of } \\
\text { the network, by continuously } \\
\text { evaluating known routes and } \\
\text { attempts to discover new } \\
\text { routes }\end{array}$ & $\begin{array}{l}\text { Optimized Link State Routing } \\
\text { (OLSR), Fish-eye State Routing } \\
\text { (FSR), Destination Sequenced } \\
\text { Distance Vector (DSDV), CGSR }\end{array}$ & $\begin{array}{l}\text { Due to increased mobility traf- } \\
\text { fic overhead is generated while } \\
\text { evaluating unnecessary routes }\end{array}$ \\
\hline & $\begin{array}{l}\text { Reactive } \\
\text { Protocol }\end{array}$ & $\begin{array}{l}\text { These are on-demand } \\
\text { protocols unlike pro-active } \\
\text { protocols only start a route } \\
\text { discovery procedure when- } \\
\text { ever needed }\end{array}$ & $\begin{array}{l}\text { AODV, DSR protocol, TORA, } \\
\text { Associativity Based Routing } \\
\text { (ABR), Signal Stability-Based } \\
\text { Adaptive Routing (SSA), } \\
\text { Location-Aided Routing } \\
\text { Protocol (LAR) }\end{array}$ & $\begin{array}{l}\text { Intermediate nodes can lead to } \\
\text { inconsistent Routes }\end{array}$ \\
\hline & $\begin{array}{l}\text { Hybrid } \\
\text { Protocol }\end{array}$ & $\begin{array}{l}\text { They combine the advantage } \\
\text { of both proactive and reac- } \\
\text { tive routing. }\end{array}$ & $\begin{array}{l}\text { ZRP, Wireless Ad hoc Routing } \\
\text { Protocol (WARP) }\end{array}$ & \\
\hline $\begin{array}{l}\text { Hierarchical } \\
\text { Routing }\end{array}$ & & $\begin{array}{l}\text { These are preferred as the } \\
\text { size of the wireless network } \\
\text { increases. }\end{array}$ & HSR, ZRP, CGSR, LANMAR & \\
\hline $\begin{array}{l}\text { Geographic } \\
\text { Position Assisted } \\
\text { Routing }\end{array}$ & & $\begin{array}{l}\text { These protocols prevent } \\
\text { network wide search for } \\
\text { destinations. }\end{array}$ & Geo-Cast, DREAM, GPSR & $\begin{array}{l}\text { The disadvantage is that all } \\
\text { nodes must have access to } \\
\text { their geographical coordi- } \\
\text { nates all the time to make the } \\
\text { geographical routing protocols } \\
\text { useful }\end{array}$ \\
\hline
\end{tabular}




\section{TCP in Mobile Ad Hoc Networks}

TCP was one of the primary protocols of internet which was originated in the beginning of network implementation. As the network is fixed, interruption and Route failures are very common. So, the loss of packets, where TCP recognizes it as timeout, leads to network congestion TCP cannot distinguish between congestion and loss of packets due to errors of transmission or failure of routes. It leads to performance degeneration in MANETs.

In MANET, losses of packets are very common in the wireless medium. Using steady link layer protocols the packet loss can be decreased. The problem of failure of route is very common which depends on the mobility of MHs. When the mobility of a Mobile Host is validating a particular route, the basic routing protocol which is reestablishing the route will take some time. Meanwhile in this time, by using current route none of the packets will reach the destination 2 . This will result in queuing of packets and desirable packet loss/acknowledgements. This will lead to source time outs which was interpreted as congestion by transport protocol. As the time out occurs, the source resends the packets which are unacknowledged. After receiving those packets the intermediate nodes which are present in the network starts congestion control algorithm which results in decrease of size of the window and eventually leads to decreasing the rate of transmission 1 . Retransmission of packets misuses limited battery power of Mobile Host and limited bandwidth. The different layers of TCP model with their description are shown in Figure 2.

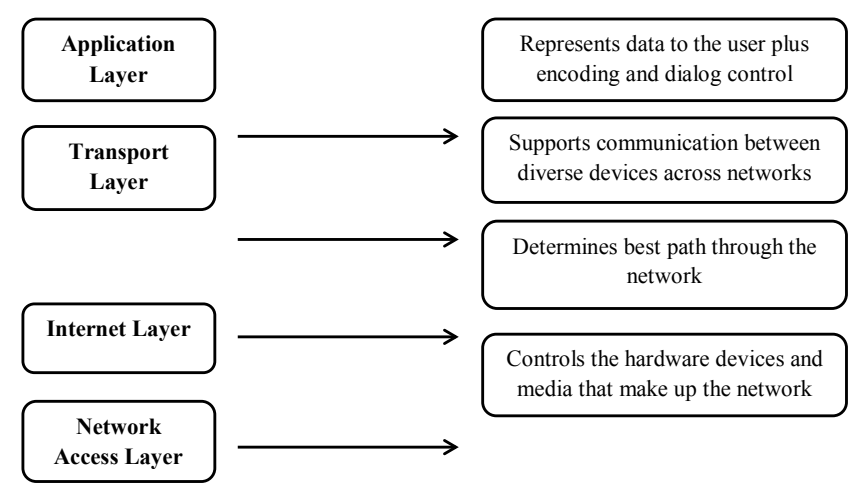

Figure 2. STCP/IP model with each layer description.

\section{Congestion Control in MANET}

To allocate and preserve resources of network reasonably is a major issue among a collection of users. The resources which are shared mostly are the queues on the routers or switches and the bandwidth of the links. In queues, Packets are queued waiting for transmission When more number of packets was going after for the same link, the packets have to be discarded and the queue will be overflowed. The network is congested when such packet drops are commonf. Since there is no fixed infrastructure in the Ad-hoc networks there are no independent routers, therefore mobile nodes act as routers. The method of Congestion control is host/node centric and router centric. In present congestion control methods, the source was informed about the network congestion. Based on the information, source will slow down the rate of transmission of packets or it may search another route which will not be best route開. As they use TCP, it is noted thatthe method of congestion control must update about the congestion problem to the source.

\section{Feedback Based Approach}

Assuming the failure of route as the congestion and requesting congestion control algorithm was mostly not preferable. Because, route failure and congestion control are needed which are to taken care handled separately and independently. So a scheme will be proposed in which the failure of route was intimated to the source so that it won't request congestion control. The source will discard sending data packets and it will start sending packets when the route was renewed 1 . Therefore a scheme called Feedback based TCP is proposed to handle failure of routes in Mobile ad-hoc networks. In Feedback based TCP, an intermediate node, upon disclosure of break of path, emerges a packet which indicates the failure of the route which is also called RFN Packet. This RFN Packet was transmitted toward the sender of TCP session.

TCP Feedback allows the Congestion control algorithm of TCP to be more effective when the sender is not in the snooze state, thereby making much more sensitive to network congestion 1 .TCP Feedback gives a simple solution to reduce the problems which are occurring due to regular path breaks MANETs. In the 
same time, it will allow the congestion control algorithm of TCP to acknowledge to network congestion 19. Feedback based TCP depends on the capability of the intermediate nodes for recognizing failure of route and the routing protocols capacity to restore a broken path within a fairly less time. Also the Failure Point (FP) should retrieve the correct path to the TCP Feedback Sender to send the RFN Packet. Feedback based TCP with nodes A, B, C, D with their states are shown in Figure 3.

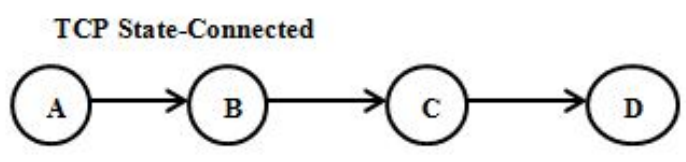

(a) TCP-F Connection from A to D

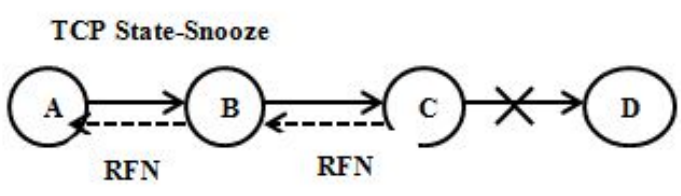

(b) Link C-D breaks and C originates RFN

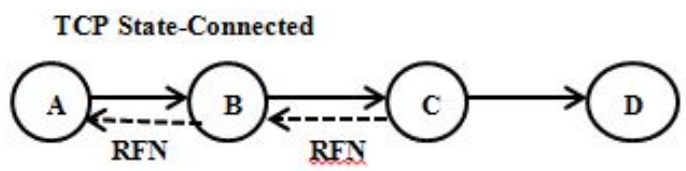

(c) Link C-D rejoins and C originates $\mathrm{RRN}$

Figure 3. Feedback based TCP with nodes A, B, and C, D.

\section{Conclusion}

The effects of failures of routes which are characteristics of MANETs Performance of TCP are studied. We have proposed a scheme which is called TCP-F or Feedback based TCP. In this approach, the source of failures of routes and reestablishments of routes are informed by failure point thus discriminate between failure of route and network congestion and to handle failure of route in MANETs. The methodology will increase the throughput, Good-put and reduce the Round trip delay time. The proposed approach will reduce the degeneration of the performance. As the overall delay will be reduced so TCP-F is expected to perform better than the basic TCP.

\section{Result}

In this paper, we have proposed a design called Feedback based TCP (TCP-F) for improving the overall TCPperformance. Various performance metrics like Throughput, Good-put, Bandwidth, Delay, Packet Delivery Ratio, Round Trip delay time, Speed of data transmission will be measured and it will be expected that Feedback based TCP or TCP with Feedback gives better performance over TCP without feedback.

\section{References}

1. Hanbali A, Altman E, Nain P. A survey of TCP over Ad hoc networks. INRIA Sophia Antipolis Cedex, France; 2005 Jun.

2. Jianli GUO, Hongwei LIU, Jian DONG, Xiaozong YANG. HEAD: A hybrid mechanism to enforce node cooperation in mobile ad hoc networks. Tsinghua Science and Technology. 2007 Jul; 12(S1):202-7.

3. Shivashankar, Suresh HN, Varaprasad G, Jayanthi G. Designing energy routing protocol with power consumption optimization in MANET. IEEE Transactions on Emerging Topics in Computing Digital Object Identifier.2013 Oct 27; 2(2):192-7. DOI:10.1109/TETC.2013.2287177.

4. Alwadiyeh ES, Aburumman AFA. Interference-aware multipath routing protocols for mobile ad hoc networks. 13th Annual IEEE Workshop on Wireless Local Networks, IEEE; 2013. p. 980.

5. Guo S, Member IEEE, Dang C, Senior Member IEEE, Yang Y, Fellow. IEEE. Joint optimal data rate and power allocation in lossy mobile ad hoc networks with delay-constrained traffics. IEEE Transactions on Computers. 2015 Mar; 64(3).

6. Chandran K, Raghunathan S, Venkatesan S, Prakash R. A feedback based scheme for improving TCP performance in ad-hoc wireless networks [Computer Science thesis]. University of Texas at Dallas Richardson, TX 75083-0688.

7. Holland G, Vaidya N. Analysis of TCP performance over mobile ad hoc networks. MobiCom '99. Proceedings of the 5th annual ACM/IEEE International Conference on Mobile Computing and Networking. Seattle, Washington, USA; 1999 Aug 15-19. p. 219-30.

8. Pacifico D, Pacifico M, Fischione C, Hjalrmasson H, Johansson $\mathrm{KH}$. Improving TCP performance during the intra LTE handover. IEEE Global Communications Conference (GLOBECOM); 2009. 
9. Abdulsaheb GM, IbrahemKhalaf O, Sulaiman N, Zmezm HF, Zmezm H. Improving ad hoc network performance by using an efficient cluster based routing algorithm. International Journal of Science and Technology. 2015 Nov; 8(30):1-8.

10. Prema A, Prabhu SJ. Enhanced in cast congestion control TCP in wireless sensor networks. International Journal of Science and Technology. 2015 Nov; 2(4).

11. Allman M, Glover D, Sanchez L. Enhancing TCP over satellite channels using standard mechanisms," RFC 2488; 1999 Jan.

12. J. Heo. Improving the start-up behavior of a congestion control scheme for TCP. In Proceedings of ACM SIGCOMM, CA, USA; 1996 Aug.

13. Yang L, Seah W, Yin Q. Improving fairness among TCP flows crossing wireless ad hoc and wired networks, In the proceedings of ACM MOBIHOC, Annapolis, Maryland, USA; 2003 Jun. p. 57-63.

14. Cordeiro C, Das S, Agarwal D. COPAS: Dynamic contention-balancing to enhance the performance of TCP over multi-hop wireless networks. In the Proceedings of IC3N, Miami, USA; 2003 Oct. p. 382-7.

15. Kopparty S, Krishnamurthy S, Faloutous M, Tripathi S. Split TCP for mobile ad hoc networks. In the Proceedings of IEEE Global Communications Conference (GLOBECOM), Taipei, Taiwan; 2002 Nov.

16. Chen K, Xue Y, Nahrstedt K. On setting TCP's congestion window limit in mobile Ad Hoc networks. In the Proceedings of IEEE International Conference on Communications (ICC), Anchorage, Alaska, USA; 2003 May.

17. Xu K, Gerla M, Qi L, Shu Y. Enhancing TCP fairness in ad hoc wireless networks using neighborhood red. In the Proceedings of ACM MOBICOM, San Diego, CA, USA; 2003 Sep. p. 16-28.

18. Gorantala K. Routing protocols in mobile ad-hoc networks [Masters Thesis]. Department of Computer Science, Umea University; 2006 Jun 15. 